\title{
Randomized Fully Dynamic Graph Algorithms with Polylogarithmic Time per Operation
}

\author{
MONIKA R. HENZINGER \\ Google, Inc., Mountain View, California
}

\author{
AND \\ VALERIE KING \\ University of Victoria, Victoria, BC, Canada
}

\begin{abstract}
This paper solves a longstanding open problem in fully dynamic algorithms: We present the first fully dynamic algorithms that maintain connectivity, bipartiteness, and approximate minimum spanning trees in polylogarithmic time per edge insertion or deletion. The algorithms are designed using a new dynamic technique that combines a novel graph decomposition with randomization. They are Las-Vegas type randomized algorithms which use simple data structures and have a small constant factor.

Let $n$ denote the number of nodes in the graph. For a sequence of $\Omega\left(m_{0}\right)$ operations, where $m_{0}$ is the number of edges in the initial graph, the expected time for $p$ updates is $O\left(p \log ^{3} n\right)$ (Throughout the paper the logarithms are base 2.) for connectivity and bipartiteness. The worst-case time for one query is $O(\log n / \log \log n)$. For the $k$-edge witness problem ("Does the removal of $k$ given edges disconnect the graph?") the expected time for $p$ updates is $O\left(p \log ^{3} n\right)$ and the expected time for $q$ queries is $O\left(q k \log ^{3} n\right)$. Given a graph with $k$ different weights, the minimum spanning tree can be maintained during a sequence of $p$ updates in expected time $O\left(p k \log ^{3} n\right)$. This implies an algorithm to maintain a $1+\epsilon$-approximation of the minimum spanning tree in expected time $O\left(\left(p \log ^{3} n \log \right.\right.$ $U) / \epsilon$ ) for $p$ updates, where the weights of the edges are between 1 and $U$.

Categories and Subject Descriptors: E.1 [Data]: Data Structures-graphs and networks; G.2.2 [Discrete Mathematics]: Graph Theory—graph algorithms
\end{abstract}

General Terms: Algorithms

Additional Key Words and Phrases: Connectivity, dynamic graph algorithms

The work of M. R. Henzinger was supported by an NSF CAREER Award and was done while at the Department of Computer Science, Cornell University, Ithaca, NY.

The research of V. King was supported by an NSERC Grant.

Authors' present addresses: M. R. Henzinger, Google, Inc., 2400 Bayshore Parkway, Mountain View, CA 94043, e-mail: monika@google.com; V. King, Department of Computer Science, University of Victoria, Victoria, BC, Canada, e-mail: val@csr.uvic.ca.

Permission to make digital/hard copy of part or all of this work for personal or classroom use is granted without fee provided that the copies are not made or distributed for profit or commercial advantage, the copyright notice, the title of the publication, and its date appear, and notice is given that copying is by permission of the Association for Computing Machinery (ACM), Inc. To copy otherwise, to republish, to post on servers, or to redistribute to lists, requires prior specific permission and/or a fee.

(C) 1999 ACM 0004-5411/99/0700-0502 \$05.00 


\section{Introduction}

In many areas of computer science, graph algorithms play an important role: Problems modeled by graphs are solved by computing a property of the graph. If the underlying problem instance changes slightly, algorithms are needed that quickly compute the property in the modified graph. Algorithms that make use of previous solutions and, thus, solve the problem faster than recomputation from scratch are called fully dynamic graph algorithms. To be precise, a fully dynamic graph algorithm is a data structure that supports the following three operations: (1) insert an edge $e$, (2) delete an edge $e$, and (3) test if the graph fulfills a certain property, for example, if two given vertices are connected.

1.1. Previous Work. In recent years a lot of work has been done in fully dynamic algorithms. ${ }^{1}$ There is also a large body of work for restricted classes of graphs and for insertions-only algorithms. The best previous time bounds for fully dynamic algorithms in undirected $n$-node graphs are: $O(\sqrt{n})$ per update for a minimum spanning forest [Eppstein et al. 1997]; $O(\sqrt{n})$ per update and $O(1)$ per query for connectivity [Eppstein et al. 1997]; $O(\sqrt{n} \log n)$ per update and $O\left(\log ^{2} n\right)$ per query for cycle-equivalence ("Does the removal of the given 2 edges disconnect the graph?") [Henzinger 1994]; $O(\sqrt{n})$ per update and $O(1)$ per query for bipartiteness ("Is the graph bipartite?") [Eppstein et al. 1997].

There is a lower bound in the cell probe model of $\Omega(\log n / \log \log n)$ on the amortized time per operation for all these problems which applies to randomized algorithms [Henzinger and Fredman 1998; Henzinger 1994; Miltersen et al. 1994]. If deletions are restricted to "undo" previous insertions in the reverse order in which the insertions occurred, then Westbrook and Tarjan [1989] gave an algorithm with takes time $O(\log n / \log \log n)$ per update. In Alberts and Henzinger [1998], it is shown that the average update time of (a variant of) the above connectivity and bipartiteness algorithms is $O(n / \sqrt{m}+\log n)$ if the edges used in updates are chosen uniformly from a given edge set. Thus, for dense graphs their average performance nearly matches the lower bound.

In plane graphs, fully dynamic algorithms for minimum spanning forest and connectivity are given in Eppstein et al. [1996] that are close to the lower bound: they take time $O\left(\log ^{2} n\right)$ per deletion and $O(\log n)$ per insertions and query. However, the constant factor of these algorithms is quite large [Eppstein et al. 1997]. Thus, the following questions were posed as open questions in Eppstein et al. [1996; 1997].

(1) Can the above properties be maintained dynamically in polylogarithmic time in (general) graphs?

(2) Is there a fully dynamic algorithm with small constants such that an efficient implementation is possible?

1.2. New Results. This paper gives a positive answer to both questions. It presents a new technique for designing fully dynamic algorithms with polylogarithmic time per operation and applies this technique to the fully dynamic

\footnotetext{
${ }^{1}$ See Alberts and Henzinger [1998], Eppstein et al. [1997], Even and Shiloach [1991], Frederickson [1985; 1997], Galil and Italiano [1992], Henzinger [1994; 1995; 1999], Henzinger and La Poutré [1995], and Spira and Pan [1975] for connectivity-related work in undirected graphs.
} 
connectivity, bipartiteness, $1+\epsilon$-approximate minimum spanning trees, and cycle-equivalence problem. The resulting algorithms are Las-Vegas type randomized algorithms which use simple data structures and have a small constant factor. They use $O(m+n \log n)$ space.

For a sequence of $\Omega\left(m_{0}\right)$ update operations, where $m_{0}$ is the number of edges in the initial graph the following amortized expected update times and worst-case query times are achieved:

(1) connectivity in update time $O\left(\log ^{3} n\right)$ and query time $O(\log n / \log \log n)$;

(2) bipartiteness in update time $O\left(\log ^{3} n\right)$ and query time $O(1)$;

(3) minimum spanning tree of a graph with $k$ different weights in update time $O\left(k \log ^{3} n\right)$;

Apart from answering queries of the form "Are nodes $u$ and $v$ connected?" the connectivity algorithm can easily be adapted to output all $l$ nodes in the connected component of a given node $u$ in time $O(\log n / \log \log n+l)$.

As an immediate consequence of these results, we achieve faster fully dynamic algorithms for the following problems:

(1) An algorithm to maintain a $1+\epsilon$-approximation of the minimum spanning tree in expected time $O\left(\left(p \log ^{3} n \log U\right) / \epsilon\right)$ for $p$ updates, where the weights of the edges are between 1 and $U$.

(2) An algorithm for the $k$-edge witness problem ("does the removal of the given $k$ edges disconnect the graph?") in update time $O\left(\log ^{3} n\right)$ and amortized expected query time $O\left(k \log ^{3} n\right)$. Note that cycle-equivalence is equivalent to the 2-edge witness problem.

(3) A fully dynamic algorithm for maintaining a maximal spanning forest decomposition of order $k$ of a graph in time $O\left(k \log ^{3} n\right)$ per update by keeping $k$ fully dynamic connectivity data structures.

A maximal spanning forest decomposition of order $k$ is a decomposition of a graph into $k$ edge-disjoint spanning forests $F_{1}, \ldots, F_{k}$ such that $F_{i}$ is a maximal spanning forest of $G_{i}=G \backslash \cup_{j<i} F_{j}$. The maximal spanning forest decomposition is interesting since $\cup_{i} F_{i}$ is a graph with $O(k n)$ edges that has the same $k$-edge connected components as $G$ [Nagamochi and Ibaraki 1992]. Subsequently the running time of our algorithms has been improved by a factor of $O(\log n)$ by using a different sampling routine [Henzinger and Thorup 1997]. Very recently, deterministic fully-dynamic graph algorithms were given with amortized time per operation of $O\left(\log ^{2} n\right)$ for connectivity and $O\left(\log ^{4} n\right)$ for minimum spanning forest, 2-edge connectivity, and biconnectivity [Holm et al. 1998].

1.3. MAIN IDEA. The new technique is a combination of a novel decomposition of the graph and randomization. The edges of the graph are partitioned into $O(\log n)$ levels such that edges in highly-connected parts of the graph (where cuts are dense) are on lower levels than those in loosely-connected parts (where cuts are sparse). For each level $i$, a spanning forest is maintained for the graph whose edges are in levels $i$ and below. If a tree edge is deleted at level $i$, we sample edges on level $i$ such that with high probability either (1) we find an edge reconnecting the two subtrees or (2) the cut defined by the deleted edge is too 
sparse for level $i$. In Case (1), we found a replacement edge fast; in Case (2), we copy all edges on the cut to level $i+1$ and recurse on level $i+1$.

We use an Eulerian tour representation of the spanning trees to linearly order the nontree edges of the graph. The Eulerian tour representation of trees was introduced in Tarjan and Vishkin [1985] and also used in Miltersen et al. [1994].

This paper is structured as follows: Section 2 gives the fully dynamic connectivity algorithm, Section 3 presents the results for $k$-weight minimum spanning trees, $1+\epsilon$-approximate minimum spanning trees, and bipartiteness.

\section{A Randomized Connectivity Algorithm}

\subsection{A Deletions-Only Connectivity Algorithm}

2.1.1. Definitions and Notation. Let $G=(V, E)$ with $|V|=n$ and $|E|=m$. We use the convention that elements in $V$ are referred to as vertices. Let $l=\lfloor\log$ $m-\log \log n\rfloor+1$. The edges of $G$ are partitioned into $l$ levels $E_{1}, \ldots, E_{l}$ such that $\cup_{i} E_{i}=E$, and for all $i \neq j, E_{i} \cap E_{j}=\emptyset$. For each $i$, we keep a forest $F_{i}$ of tree edges such that $F_{i}$ is a spanning forest of $\left(V, \cup_{j \leq i} E_{j}\right)$. So, for $i>1$, $F_{i-1} \subseteq F_{i}$ and $F_{i} \backslash F_{i-1} \subset E_{i}$. Then, $F_{l}$ is a spanning tree of $G$ and edges in $E \backslash F$ are referred to as nontree edges. A spanning tree $T$ on level $i$ is a tree of $F_{i}$.

All nontree edges incident to vertices in $T$ are stored in a data structure that is described in more detail below. The weight of $T$, denoted $w(T)$, is two times the number of nontree edges with both endpoints in $T$ plus the number of nontree edges with exactly one endpoint in $T$. The size of $T$, denoted $s(T)$, is the number of vertices in $T$. A tree is smaller than another tree if its size is no greater than the others. We say level $i$ is below level $i+1$.

2.1.2. The Algorithm. Initially, all edges are in $E_{1}$, and we compute $F_{1}$, which is a spanning tree of $G$.

When an edge $e$ is deleted, remove $e$ from the graph containing it. If $e$ is a tree edge, let $i$ be the level such that $e \in E_{i}$. Call Replace $(e, i)$.

$\operatorname{Replace}(e, i)$.

Let $T$ be the level $i$ tree containing edge $e$ and let $T_{1}$ and $T_{2}$ be the two subtrees of $T$ that resulted from the deletion of $e$, such that $s\left(T_{1}\right) \leq s\left(T_{2}\right)$.

- If $w\left(T_{1}\right) \leq \log ^{2} n$ goto Case 2 .

- Sample. We sample $c \log ^{2} m$ nontree edges of $E_{i}$ incident to vertices of $T_{1}$ for some appropriate constant $c$. An edge with both endpoints in $T_{1}$ is picked with probability $2 / w\left(T_{1}\right)$ and an edge with one endpoint in $T_{1}$ is picked with probability $1 / w\left(T_{1}\right)$.

- Case 1: Replacement edge found. If one of the sampled edges connects $T_{1}$ to $T_{2}$ then add it to all $F_{j}, j \geq i$.

- Case 2: Sampling unsuccessful. If none of the sampled edges connects $T_{1}$ and $T_{2}$, search all edges incident to $T_{1}$ and determine $S=$ \{nontree edges connecting $T_{1}$ and $\left.T_{2}\right\}$.

- If $|S|>w\left(T_{1}\right) /\left(15 c^{\prime} \log n\right)$ choose one element of $S$ and add it to $F_{j}, j \geq i$.

- If $0<|S| \leq w\left(T_{1}\right) /\left(15 c^{\prime} \log n\right)$, remove the elements of $S$ from $E_{i}$, and insert them into $E_{i+1}$. Then add one of the newly inserted edges to $F_{j}, j>i$.

- If $S=\emptyset$ then if $i<l$ then do

- Replace $(e, i+1)$. Else stop. 
We show below that choosing $l=O(\log n)$ suffices since only a constant fraction of the edges in $E_{i}$ will move to $E_{i+1}$. Furthermore, we show that in Case 2 the case that $|S|>w\left(T_{1}\right) /\left(15 c^{\prime} \log n\right)$ only occurs with probability $O\left(1 / n^{2}\right)$. If this case does not arise, then the cost of Replace $(e, i)$ can be paid for by charging $O\left(\log ^{3} n\right)$ to the update operation and/or $O\left(\log ^{2} n\right)$ to the edges that are moved to $E_{i+1}$. This is possible since we give a data structure that takes time $O(\log n)$ to sample, test, insert, or delete an edge. Since each edge is moved at most $l$ times, we show that this leads to an expected total time of $O\left(m \log ^{3} n\right)$ for a sequence of $m$ deletions.

2.1.3. Proof of Correctness. We first show that all edges are contained in $\cup_{i \leq l} E_{i}$, that is, when Replace $(e, l)$ is called, and Case 2 occurs, no edges will be inserted into $E_{l+1}$. We use this fact to argue that if a replacement edge exists, it will be found.

Let $m_{i}$ be the number of edges ever in $E_{i}$.

Lemma 2.1. For all smaller trees $T_{1}$ on level $i, \sum w\left(T_{1}\right) \leq 15 m_{i} \log n$.

PROOF. We use the "bankers view" of amortization: Every edge of $E_{i}$ receives a coin whenever it is incident to the smaller tree $T_{1}$. We show that the maximum number of coins accumulated by the edges of $E_{i}$ is $15 m_{i} \log n$.

Each edge of $E_{i}$ has two accounts, a start-up account and a regular account. Whenever an edge $e$ of $E_{i}$ is moved to level $i>1$, the regular account balance of the two edges on level $i$ with maximum regular account balance is set to 0 and all their coins are paid into $e$ 's start-up account. Whenever an edge of $E_{i}$ is incident to the smaller tree $T_{1}$ in a split of $T$, one coin is added to its regular account.

We show by induction on the steps of the algorithm that a start-up account contains at most $10 \log n$ coins and a regular account contains at most $5 \log n$ coins. The claim obviously holds at the beginning of the algorithm. Consider step $k+1$. If it moves an edge to level $i$, then by induction the maximum regular account balance is at most $5 \log n$ and, thus, the start-up account balance of the new edge is at most $10 \log n$.

Consider next the case that step $k+1$ splits tree $T_{1}$ off $T$ and charges one coin to each edge $e$ of $E_{i}$ incident to $T$. Let $w_{0}$ be the weight of $T$ when $T$ was created. We show that if there exists an edge $e$ such that $e$ 's regular account balance is larger than 0 , then $w\left(T_{1}\right) \leq 3 w_{0} / 4$. This implies that at most $2 \log _{4 / 3} n<$ $5 \log n$ splits can have charged to $e$ after $e$ 's last reset. The lemma follows.

Edges incident to $T$ at its creation are reset before edges added to level $i$ later on. All edges added to level $i$ later on and incident to $T$ have a 0 regular account balance. Since $e$ has a non-zero regular account balance, at most $w_{0} / 2$ many inserts into level $i$ can have occurred since the creation of $T$. Thus, immediately before the split, $w(T) \leq 3 w_{0} / 2$. Since $w\left(T_{1}\right) \leq w(T) / 2$, the claim follows.

LEMMA 2.2. For any $i, m_{i} \leq m / c^{\prime i-1}$.

Proof. We show the lemma by induction. It clearly holds for $i=1$. Assume it holds for $E_{i-1}$. When summed over all smaller trees $T_{1}, \sum w\left(T_{1}\right) /\left(15 c^{\prime} \log n\right)$ edges are added to $E_{i}$. By Lemma 2.1, $\Sigma w\left(T_{1}\right) \leq 15 m^{\prime} \log n$ where $m^{\prime}$ is the total number of edges ever in level $i-1$. Hence, $m_{i} \leq\left(2 m_{i-1} \log n\right) /\left(2 c^{\prime} \log \right.$ $n)=m /\left(c^{\prime i-1}\right)$. 
Choosing $c^{\prime}=2$ and observing that edges are never moved to a higher level from a level with less than $2 \log n$ edges gives the following corollary.

COROLlary 2.3. For $l=\lfloor\log m-\log \log n\rfloor+1$, all edges of $E$ are contained in some $E_{i}, i \leq l$.

The following relationship, which will be useful in the running time analysis, is also evident.

Corollary 2.4. $\quad \Sigma_{i} m_{i}=O(m)$.

THEOREM 2.5. $F_{i}$ is a spanning forest of $\left(V, \cup_{j \leq i} E_{j}\right)$.

Proof. Initially, this is true, since we compute $F_{1}$ to be the spanning tree of $E=E_{1}$.

Consider the first time it fails, when a tree edge $e$ is deleted, and a replacement edge exists but is not found. By Corollary 2.3, the replacement edge lies in some $E_{i}, i \leq l$.

Let $i$ be the minimum level at which a replacement edge $r, s$ exists. Let $e \in$ $E_{k}$. We claim $i \geq k$. Since $r$ and $s$ are connected in $\cup_{j \leq i} E_{j}$ and we have not failed yet, there is a path $P$ in $F_{i}$ from $r$ to $s$. Since $r, s$ is a replacement edge for $e, e \in P$. Thus, $e \in F_{i}$, and hence $i \geq k$. It follows that $\operatorname{Delete}(e, i)$ will be called.

Either a replacement edge will be found by sampling or every edge incident to $T_{1}$ will be examined. We claim that every replacement edge $\{r, s\}$ is incident to $T_{1}$. Suppose it is not. By assumption, there is a path from $r$ to $s$ in $\cup_{j \leq i} E_{j}$. If this path includes $e$, then either $r$ or $s$ is in $T_{1}$. If it doesn't include $e$, then $\{r, s\}$ forms a cycle with $F-e$ contradicting the assumption that $\{r, s\}$ is a replacement edge.

2.1.4. The Euler Tour Data Structure. In this subsection, we present the data structure that we use to implement the algorithm of the previous section efficiently. We encode an arbitrary tree $T$ with $n$ vertices using a sequence of $2 n-1$ symbols, which is generated as follows: Root the tree at an arbitrary vertex. Then call ET(root), where ET is defined as follows:

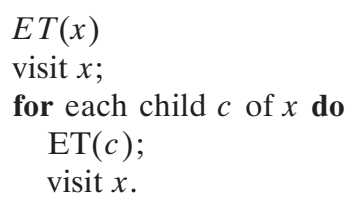

Each edge of $T$ is visited twice and every degree- $d$ vertex $d$ times, except for the root, which is visited $d+1$ times. Each time any vertex $u$ is encountered, we call this an occurrence of the vertex and denote it by $o_{u}$.

New encodings for trees resulting from splits and joins of previously encoded trees can easily be generated. Let $E T(T)$ be the sequence representing an arbitrary tree $T$.

\section{Procedures for modifying encodings}

(1) To delete edge $\{\boldsymbol{a}, \boldsymbol{b}\}$ from $T$ : Let $T_{1}$ and $T_{2}$ be the two trees that result, where $a \in$ $T_{1}$ and $b \in T_{2}$. Let $o_{a_{1}}, o_{b_{1}}, o_{b_{2}}$ represent the occurrences encountered in the two 
traversals of $\{a, b\}$. If $o_{a_{1}}<o_{b_{1}}$ and $o_{b_{1}}<o_{b_{2}}$, then $o_{a_{1}}<o_{b_{1}}<o_{b_{2}}<o_{a_{2}}$. Thus, $E T\left(T_{2}\right)$ is given by the interval of $E T(T) o_{b_{1}}, \ldots, o_{b_{2}}$ and $E T\left(T_{1}\right)$ is given by splicing out of $E T(T)$ the sequence $o_{b_{1}}, \ldots, o_{a_{2}}$.

(2) To change the root of $\boldsymbol{T}$ from $\boldsymbol{r}$ to $s$ : Let $o_{s}$ denote any occurrence of $s$. Splice out the first part of the sequence ending with the occurrence before $o_{s}$, remove its first occurrence $\left(o_{r}\right)$, and tack the first part on to the end of the sequence, which now begins with $o_{s}$. Add a new occurrence $o_{s}$ to the end.

(3) To join two rooted trees $\boldsymbol{T}$ and $\boldsymbol{T}^{\prime}$ by edge $e$ : Let $e=\{a, b\}$ with $a \in T$ and $b \in T^{\prime}$. Given any occurrences $o_{a}$ and $o_{b}$, reroot $T^{\prime}$ at $b$, create a new occurrence $o_{a_{n}}$ and splice the sequence $E T\left(T^{\prime}\right) o_{a_{n}}$ into $E T(T)$ immediately after $o_{a}$.

If the sequence $E T(T)$ is stored in a balanced search tree of degree $b$, and height $O(\log n / \log b)$ then one may insert an interval or splice out an interval in time $O(b \log n / \log b)$, while maintaining the balance of the tree, and determine if two elements are in the same tree, or if one element precedes the other in the ordering in time $O(\log n / b)$.

Aside from lists and arrays, the only data structures used in the connectivity algorithm are trees represented as sequences which are stored in balanced $b$-ary search trees. We next describe these data structures.

2.1.4.1. Data StRuctures. For each spanning tree $T$ on each level $i<l$, each occurrence of $E T(T)$ is stored in a node of a balanced binary search tree we call the $E T(T)$-tree. For each tree $T$ on the last level $l, E T(T)$ is stored in a balanced $(\log n)$-ary search tree. Note that there are no nontree edges on this level. For each vertex $u \in T$, we arbitrarily choose one occurrence to be the active occurrence of $u$.

With the active occurrence of each vertex $v$, we keep an (unordered) list of nontree edges in level $i$ which are incident to $v$, stored as an array. Each node in the ET-tree contains the number of nontree edges and the number of active occurrences stored in its subtree. Thus, the root of $E T(T)$ contains the weight and size of $T$.

In addition to storing $G$ and $F$ using adjacency lists, we keep some arrays and lists:

-For each vertex and each level, a pointer to the vertex's active occurrence on that level.

-For each tree edge, for each level $k$ such that $e \in F_{k}$, pointers to each of the four (or three, if an endpoint is a leaf) occurrences associated with its traversal in $F_{k}$;

-For each nontree edge, pointers to the locations in the two lists of nontree edge containing the edge and the reverse pointers.

-For each level $i$, a list containing a pointer to each root of a $E T(T)$-tree, for all spanning trees $T$ at level $i$, and for each root a pointer back to the list;

-For each level $i$, a list of tree edges in $E_{i}$ and for each edge a pointer back to its position in the list.

2.1.5. Implementation. Using the data structures described above, the following operations can be executed on each spanning tree on each level. Let $T$ be a spanning tree on level $i$. 
- tree $(x, i)$ : Return a pointer to $E T(T)$ where $T$ is the spanning tree of level $i$ that contains vertex $x$.

—nontree_edges $(T)$ : Return a list of nontree edges stored in $E T(T)$; each edge is returned once or twice.

- sample\&test $(T)$ : Randomly select a nontree edge of $E_{i}$ that has at least one endpoint in $T$, where an edge with both endpoints in $T$ is picked with probability $2 / w(T)$ and an edge with exactly one endpoint in $T$ is picked with probability $1 / w(T)$. Test if exactly one endpoint is in $T$, and if so, return the edge.

-insert_tree $(e, i)$ : Join by $e$ the two trees on level $i$, each of which contains an endpoint of $e$.

-delete_tree $(e, i)$ : Remove $e$ from the tree on level $i$ which contains it.

-insert_nontree $(e, i)$ : Insert the nontree edge $e$ into $E_{i}$.

-delete_nontree $(e)$ : Delete the nontree edge $e$.

The following running times are achieved using a binary search tree: tree, sample\&test, insert_non_tree, delete_non_tree, delete_tree, and insert_tree in $O(\log$ $n$ ) and nontree_edges $(\bar{T})$ in $O\left(m^{\prime} \log \bar{n}\right)$, where $\bar{m}^{\prime}$ is the number of returned edges. On the last level $l$, when a $(\log n)$-ary tree is used, the running time of delete_tree and insert tree is increased to $O\left(\log ^{2} n / \log \log n\right)$ and the running time of tree is reduced to $O(\log n / \log \log n)$. We describe the implementation details of these operations.

$\operatorname{tree}(x, i)$ : Follow the pointer to the active occurrence of $x$ at level $i$. Traverse the path in the $E T(T)$-tree from the active occurrence to the root and return a pointer to this root.

nontree_edges $(T)$ : Traverse all nodes of $E T(T)$ with incident non-tree edges and output every nonempty list of nontree edges encountered at a node.

sample\&test $(T)$ : Let $T$ be a level $i$ tree. Pick a random number $j$ between 1 and $w(T)$ and find the $j$ th nontree edge $\{u, v\}$ stored in the $E T(T)$. If $\operatorname{tree}(u, l) \neq$ $\operatorname{tree}(v, l)$, then return the edge.

insert_tree $(e, i)$ : Determine the active occurrences of the endpoints of $e$ on level $\bar{i}$ and follow Procedure 3 for joining two rooted trees, above. Update pointers to the root of the new tree and the list of tree edges on level $i$.

delete_tree $(e, i)$ : Let $e=\{u, v\}$. Determine the four occurrences associated with the traversal of $e$ in the tree on level $i$ which contains $e$ and delete $e$ from it, following Procedure 1, above. Update pointers to the roots of the new trees, the list of tree edges, and (if necessary) the active occurrences of $u$ and $v$.

insert_nontree $(e, i)$ : Determine the active occurrences of the endpoints of $e$ on level $i$ and add $e$ to the list of edges stored at them.

delete_nontree $(e)$ : Follow the pointers to the two locations of $e$ in lists of non-tree edges and remove $e$.

Using these functions, the deletions-only algorithm can be implemented as follows: 


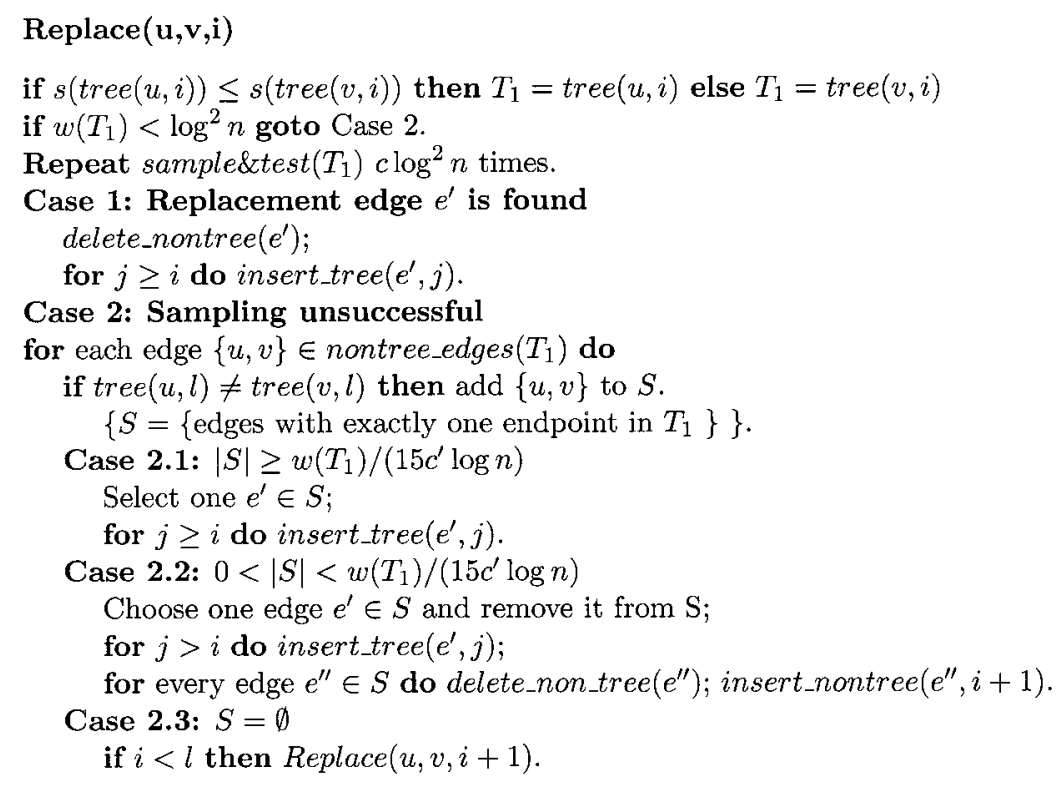

FIG. 1.

To initialize the data structures: Given a graph $G$ compute a spanning forest of $G$. Compute the $E T(T)$ for each $T$ in the forest, select active occurrences, and set up pointers as described above. Initially, the set of trees is the same for all levels. Then insert the nontree edges with the appropriate active occurrences into level 1 and compute the number of nontree edges in the subtree of each node.

To answer the query: "Are $\boldsymbol{x}$ and $\boldsymbol{y}$ connected?": Test if tree $(x, l)=\operatorname{tree}(y, l)$.

To update the data structure after a deletion of edge $e=\{u, v\}$ : If $e$ is in $E_{i}$, then do delete_tree $(e, j)$ for $j \geq i$, and call Replace $(u, v, i)$.

If $e$ is not a tree edge, execute delete nontree $(e)$. (See Figure 1.)

In the deletions-only case, we choose $c=8$ and $c^{\prime}=2$, in the fully dynamic case we choose $c=16$ and $c^{\prime}=4$.

2.1.6. Analysis of Running Time. We show that the amortized cost per deletion is $O\left(\log ^{3} n\right)$ if there are $m$ deletions.

In all cases where a replacement edge is found, $O(\log n)$ insert_tree operations are executed, costing $O\left(\log ^{2} n\right)$. In addition:

Case 1: Sampling is successful. The cost of sample\&test is $O(\log n)$ and this is repeated $O\left(\log ^{2} n\right)$ times, for a total of $O\left(\log ^{3} n\right)$.

Case 2: Sampling is not successful or $w\left(T_{1}\right)<\log ^{2} n$. We refer to the executing nontree_edges $\left(T_{1}\right)$ and the testing of each edge as the cost of gathering and testing the nontree edges. The first operation $\operatorname{costs} O(\log n)$ per nontree edge and the second is $O(\log n / \log \log n)$ per nontree edge, for a total cost of $O\left(w\left(T_{1}\right)\right.$ $\log n)$. Now there are three possible cases.

Case 2.1: $|S| \geq w\left(T_{1}\right) /\left(15 c^{\prime} \log n\right)$. If $w\left(T_{1}\right)<\log ^{2} n$, we charge the cost of gathering and testing to the delete operation. Otherwise, the probability of this subcase occurring is $\left(1-1 /\left(15 c^{\prime} \log n\right)\right)^{c} \log ^{2} n=O\left(1 / n^{2}\right)$ for $c=30 c^{\prime}$, and 
the total cost of this case is $O\left(w\left(T_{1}\right) \log n\right)$. Thus this contributes an expected cost of $O(\log n)$ per operation.

Cases 2.2 and 2.3: $|S|<w\left(T_{1}\right) /\left(15 c^{\prime} \log n\right)$. Each delete_nontree, insert_nontree, and insert_tree costs $O(\log n)$, for a total cost of $O\left(w\left(\bar{T}_{1}\right) \log n\right)$. In this case, $\operatorname{tree}(u, i)$ and tree $(v, i)$ are not reconnected. Note that only edges incident to the smaller tree $T_{1}$ are gathered and tested. Thus, over the course of the algorithm, the cost incurred in these cases on any level $i$ is $O\left(\sum w\left(T_{1}\right) \log n\right)$, where the sum is taken over all smaller trees $T_{1}$ on level $i$. From Lemma 2.1, we have the $\sum w\left(T_{1}\right) \leq 15 m_{i} \log n$. From Corollary 2.4, $\Sigma_{i} m_{i}=O(m)$, giving a total cost of $O\left(m \log ^{2} n\right)$.

\subsection{A Fully Dynamic Connectivity Algorithm}

2.2.1. The Algorithm. Next, we also consider insertions. When an edge $\{u, v\}$ is inserted into $G$, add $\{u, v\}$ to $E_{l}$. If $u$ and $v$ were not previously connected in $G$, that is, $\operatorname{tree}(u, l) \neq \operatorname{tree}(v, l)$, add $\{u, v\}$ to $F_{l}$.

We let the number of levels be $l=\lceil 2 \log n\rceil$. A rebuild of the data structure is executed periodically. A rebuild of level $i$, for $i \geq 1$, is done by a move_edges $(i)$ operation, which moves all tree and nontree edges in $E_{j}$ for $j>i$ into $\bar{E}_{i}$. Also, for each $j>i$, all tree edges in $E_{j}$ are inserted into all $F_{k}, j<k \leq i$. Note that after a rebuild at level $i, E_{j}$, for $j>i$, contains no edges, and $F_{j}=F_{i}$ for all $j \geq$ $i$, that is, the spanning trees on level $j \geq i$ span the connected components of $G$.

After each insertion, we increment $I$, the number of insertions modular $2^{\lceil 2 \log n\rceil}$ since the start of the algorithm. Let $j$ be the greatest integer $k$ such that $2^{k} \mid I$. After an edge is inserted, a rebuild of level $l-j-1$ is executed. If we represent $I$ as a binary counter whose bits are $b_{0}, \ldots, b_{l-1}$, where $b_{0}$ is the most significant bit, then a rebuild of level $i$ occurs each time the $i$ th bit flips to 1 .

2.2.2. Proof of Correctness. The proof of correctness is the same as the one for the deletions-only case, except that we must set the value of $l$ to $\lceil 2 \log n\rceil$ and alter the argument which shows that all edges are contained in $\cup_{i \leq l} E_{i}$.

We define an $i$-period to be any period beginning right after a rebuild of level $j \leq i$, or the start of the algorithm and ending right after the next rebuild of level $j^{\prime} \leq i$. That is, an $i$-period starts right after the flip to 1 of some bit $j \leq i$, or the start of the algorithm and ends with next such flip. Note that there are two types of $i$-periods: (A) begins immediately after a rebuild of level $j<i$, that is, all edges from $E_{i}$ are moved to some $E_{j}$ with $j<i\left(b_{j}, j>i\right.$, flips to 1$)$; (B) begins immediately after a rebuild of level $i$, that is, all edges from $\cup_{j>i} E_{j}$ are moved into $E_{i}\left(b_{i}\right.$ flips to 1$)$.

It is easy to see that an $i$-1-period consists of two parts, one type A $i$-period, followed by one type $\mathrm{B} i$-period, since any flip to 1 by some bit $b_{j}, j<i$ must be followed by a flip of $b_{i}$ to 1 before a second bit $b_{j^{\prime}}, j^{\prime}<i$ flips to 1 .

THEOREM 2.6. Let $a_{i}$ be the number of edges in $E_{i}$ during an $i$-period. Then $a_{i}<$ $n^{2} / 2^{i-1}$.

Proof. By a proof analogous to that of Lemma 2.1, we have:

LEMMA 2.7. For all smaller trees $T_{1}$ on level $i$ that were searched between two consecutive rebuilds of levels $j, j^{\prime} \leq i, \sum w\left(T_{1}\right) \leq 15 a_{i} \log n$. 
We now bound $a_{i}$. Note that we may restrict our attention to the edges that are moved into $E_{i}$ during any one $i$-1-period since $E_{i}$ is empty at the start of each $i$-1-period.

Thus, an edge is in $E_{i}$ either because it was passed up from $E_{i-1}$ during one $i-1$-period or moved there in a single rebuild of level $i$.

Since $E_{k}$ for $k \geq i$ was empty at the start of the $i$-1-period, any edge moved to $E_{i}$ during the rebuild of level $i$ was passed up from $E_{i-1}$ to $E_{i}$ and then to $E_{i-1}$ during the type A $i$-period (i.e., the first part of the $i$-1-period) or was inserted into $G$ during the type A $i$-period. We have

$$
a_{i} \leq h_{i-1}+b_{i},
$$

where $h_{i-1}$ is the maximum number of edges passed up from $E_{i-1}$ to $E_{i}$ during a single $i$-1-period (i.e., an A $i$-period followed by a B $i$-period) and $b_{i}$ is the number of edges inserted into $G$ during a single $i$-period.

The number of edges inserted into $G$ during an $i$-period is $2^{l-i-1}$.

To bound $h_{i-1}$ we use Lemma 2.7 to bound $\Sigma w\left(T_{1}\right)$, summed over all smaller trees $T_{1}$ which are searched on level $i-1$ during an $i$-1-period. As in the proof of Lemma 2.2, we can now bound $h_{i-1}$. Choosing $c^{\prime}=4$ gives $h_{i-1} \leq a_{i-1} / 4$.

Substituting for $h_{i-1}$ and $b_{i}$ yields

$$
a_{i} \leq 2^{l-i-1}+\frac{a_{i-1}}{4} .
$$

Choosing $l=\lceil 2 \log n\rceil$ and noting that $a_{1}<n^{2}$, an induction proof shows that $a_{i}<n^{2} / 2^{i-1}$.

This implies $a_{l}<2$ and edges are never passed up to $a_{l+1}$. We have:

Corollary 2.8. For $l=\lceil 2 \log n\rceil$, all edges of $E$ are contained in some $E_{i}$, $i \leq l$.

2.2.3. Analysis of the Running Time. To analyze the running time, note that the analysis of Case 1 and Case 2.1, above, are not affected by the rebuilds. However, (1) we have to bound the cost incurred during an insertion, that is, the cost of the operation move_edges and (2) in Case 2.2 and 2.3, the argument that $O\left(m_{i} \log n\right)$ edges are gathered and tested (using nontree_edges and tree) on level $i$ during the course of the algorithm must be modified.

The cost of (1), that is, the cost of executing move_edges $(i)$ is the cost of moving each tree edge and each nontree edge in $E_{j}, j>\bar{i}$ into $E_{i}$ and the cost of updating all the $F_{k}, j<k \leq i$.

To analyze the first part, we note that each move of an edge into $E_{i}$ costs $O(\log n)$ per edge. The number of edges moved is no greater than $\sum_{j>i} a_{j}<$ $n^{2} / 2^{i}$. Thus, the cost incurred is $O\left(n^{2} \log n / 2^{i}\right)$.

The cost of inserting one tree edge into any given level is $O(\log n)$ per edge. A tree edge is added only once into a level, since a tree edge is never passed up. Thus, this cost may be charged to the edge for a total cost of $O\left(\log ^{2} n\right)$ per edge.

We analyze the cost of (2).

For $i<l$, if fewer than $2^{l-i-1}$ edges have been inserted since the start of the algorithm then no rebuilds have occurred at level $i$ or lower, and the analysis for level $i$ of the deletions-only argument holds. That is, the costs incurred on level $i$ 
is bounded above by $O\left(m \log ^{2} n / c^{\prime i}\right)$, where $m$ is the number of edges in the initial graph.

Applying Lemma 2.7, we conclude that the cost for the gathering and testing of edges from all smaller trees $T_{1}$ on level $i$ during an $i$-period is $O\left(15 a_{i} \log n * \log \right.$ $n)=O\left(n^{2} \log ^{2} n / 2^{i}\right)$.

For $i=l$, we note that since there are $O(1)$ edges in $E_{l}$ at any given time, and since the cost is $O(\log n)$ per edge for gathering and testing, the total cost for each instance of gathering and testing on this level is $O(\log n)$.

We use a potential function argument to charge the cost of (1) and (2) to the insertions. Each new insertion contributes $c^{\prime \prime} \log ^{2} n$ tokens toward the bank account of each level, for a total of $\Theta\left(\log ^{3} n\right)$ tokens. Since an $i$-period occurs every $n^{2} / 2^{i}$ insertions, the tokens contributed by these insertions can pay for the $O\left(n^{2} \log ^{2} n / 2^{i}\right)$ cost of the gathering and testing on level $i$ during the $i$-period and the $O\left(n^{2} \log n / 2^{i}\right)$ cost of move_edges $(i)$ incurred at most once during the $i$-period.

2.3. ImPROVEMENTS. In this section, we present a simple "trick" which reduces the cost of testing all nontree edges incident to a smaller tree $T_{1}$ to $O(1)$ per edge so that the total cost of gathering and testing edges incident to $T_{1}$ is $O(1)$ per edge.

2.3.1. Constant Time for Gathering and Testing. As noted above, since the nontree edges incident to an ET-tree are available as a list, the time needed to retrieve these edges is $O(1)$ per edge. One can also test each nontree edge in $O(1)$ time, that is, determine the set $S$ of all nontree edges that contain only one endpoint, by running through the list three times. For each edge in the list, initialize the $i, j$ entry of an $n \times n$ array. Then use these entries to count the number of times each edge appears in the list. Traverse the list again and add to $S$ any edge whose count is one.

2.3.2. Constant Query and Test Time for Deletions-Only Algorithms. We note that determining whether two vertices $i$ and $j$ are in the same component, that is, is tree $(i)=\operatorname{tree}(j)$, can be speeded up to $O(1)$ for the deletions-only algorithm. A component is split when Replace $(e, l)$ is called and no replacement edge is found. In that case, label the nodes of the smaller component $T_{1}$ with a new label. The cost of doing so is proportional to the size of $T_{1}$. Over the course of the algorithm, the cost is $O(n \log n)$ since each node appears in a smaller component no more than $\log n$ times. Then, tree $(i)=\operatorname{tree}(j)$ iff $i$ and $j$ have the same label.

This improvement does not affect the asymptotic running time of the randomized connectivity algorithm, as that is dominated by the cost of the random sampling.

\section{Randomized Algorithms for Other Dynamic Graph Problems}

In this section, we show that some dynamic graph problems have polylogarithmic expected update time, by reducing these problems to connectivity and we give an alternative algorithm for maintaining the minimum spanning tree. 
3.1. A k-Weight Minimum Spanning Tree Algorithm. The $k$-weight minimum spanning tree problem is to maintain a minimum spanning forest in a dynamic graph with no more than $k$ different edgeweights at any given time.

Let $G=(V, E)$ be the initial graph. Compute the minimum spanning forest $F$ of $G$. We define a sequence of subgraphs $G_{1}, G_{2}, \ldots, G_{k}$ on nodeset $V$ and with edgesets $E_{1}, E_{2}, \ldots, E_{k}$ as follows: Let $E_{i}=$ \{edges with weight of rank $i\} \cup F$. If initially, there are $l<k$ distinct edgeweights, then for $i>l$, $E_{i}=F$. These $E_{i}$ are called "extras". The spanning forests of each $G_{i}$ are maintained as in the connectivity algorithm. These forests and $F$ are also stored in dynamic trees [Sleator and Tarjan 1983]. The subgraphs are ordered by the weight of its edgeset and stored in a balanced binary tree.

To insert edge $\{u, v\}$ into $G$ : Determine if $u$ and $v$ are connected in $F$. If so, find the maximum cost edge $e$ on the path from $u$ to $v$ in $F$ (using the dynamic trees). If the weight of $e$ is greater than the weight of $\{u, v\}$, replace $e$ in $F$ by $\{u, v\}$. If $u$ and $v$ were not previously connected, add $\{u, v\}$ to $F$. Otherwise, just add $\{u, v\}$ to $E_{j}$ where $j$ is the rank of the weight of $\{u, v\}$. If $\{u, v\}$ is the only edge of its weight in $G$, then create a new subgraph by adding $\{u, v\}$ to an extra and inserting it into the ordering of the other $G_{i}$. Update the $E_{i}$ to reflect the changes to $F$.

To delete edge $\{u, v\}$ from $G$ : Delete $\{u, v\}$ from all graphs containing it. To update $F$ : If $\{u, v\}$ had been in $F$, then a tree $T$ in $F$ is divided into two components. Find the minimum $i$ such that $u$ and $v$ are connected in $G_{i}$ using binary search on the list of subgraphs. Now, search the path from $u$ to $v$ in the spanning forest of $G_{i}$ to find an edge crossing the cut in $T$. Use binary search: Let $x$ be a midpoint of the path. Recurse on the portion of the path between $u$ and $x$ if $u$ and $x$ are not connected in $F$; else recurse on the path between $x$ and $v$.

Correctness: When an edge $\{u, v\}$ is inserted and its cost is not less than the cost of the maximum cost edge on the tree path between $u$ and $v$, then the minimum spanning forest $F$ is unchanged. If the cost of $\{u, v\}$ is less than the cost of the maximum cost edge $e^{\prime}$ on the tree path between $u$ and $v$, then replacing $e^{\prime}$ by $\{u, v\}$ decreases the cost of the minimum spanning tree by the maximum possible amount and gives, thus, the minimum spanning tree of $G \cup$ $\{u, v\}$.

Analysis of Running Time: The algorithm (1) determines how $F$ has to be changed, and (2) updates the data structures.

(1) After an insertion the maximum cost edge on the tree path between $u$ and $v$ can be determined in time $O(\log n)$ using the dynamic tree data structure of $F$. After a deletion, it takes time $O\left(\log ^{2} n / \log \log n\right)$ to find the minimum $i$ such that $u$ and $v$ are connected in $G_{i}$, since a connectivity query on a level takes time $O(\log n / \log \log n)$. The midpoint of the tree path between $u$ and $v$ in $G_{i}$ can be determined in time $O(\log n)$ using the dynamic tree data structure of the spanning tree of $G_{i}$. The algorithm recurses at most $\log n$ times to determine the replacement edge for $\{u, v\}$, for a total of $O\left(\log ^{2} n\right)$.

(2) The insertion or deletion of $\{u, v\}$ into $E_{i}$, where $i$ is the rank of the weight of $\{u, v\}$, takes amortized expected time $O\left(\log ^{3} n\right)$. If $F$ changes, one 
additional insertion and deletion is executed in every $E_{j}$. For each update there are a constant number of operations in the dynamic tree of $F$ and of the spanning tree of every $E_{j}$, each $\operatorname{costing} O(\log n)$. Thus, the amortized expected update time is $O\left(k \log ^{3} n\right)$.

3.2. A $1+\epsilon$-Approximate Minimum Spanning Tree Algorithm. Given a graph with weights between 1 and $U$, a $1+\epsilon$-approximation of the minimum spanning tree is a spanning tree whose weight is within a factor of $1+\epsilon$ of the weight of the optimal. The problem of maintaining a $1+\epsilon$ approximation is easily seen to be reducible to the $k$-weight MST problem, where a weight has rank $i$ if it falls in the interval $\left[(1+\epsilon)^{i},(1+\epsilon)^{i+1}\right)$ for $i=0,1, \ldots,\lfloor\log$ $U / \log (1+\epsilon)\rfloor$. This yields an algorithm with amortized cost $O\left(\left(\log ^{3} n \log U\right) / \epsilon\right)$.

3.3. A Bipartiteness Algorithm. The bipartite graph problem is to answer the query "Is $G$ bipartite?" in $O(1)$ time, where $G$ is a dynamic graph.

We reduce this problem to the 2-weight minimum spanning tree problem. We use the fact that a graph $G$ is bipartite iff given any spanning forest $F$ of $G$, each nontree edge forms an even cycle with $F$. Call these edges "even edges" and the remaining edges "odd". We also use the fact that if an edge $e$ in $F$ is replaced with an even edge then the set of even edges is preserved. Let $C$ be the cut in $F$ induced by removing $e$. If $e$ is replaced with an odd edge then for each nontree edge $e^{\prime}$ which crosses $C$ the parity of $e^{\prime}$ changes. We replace an edge by an odd replacement edge only if there does not exists an even replacement edge. Thus, the parity of an even edge never changed. $F$ is stored as a dynamic tree.

Our algorithm is: generate a spanning forest $F$ of the initial graph $G$. All tree and even nontree edges have weight 0 . Odd edges have weight 1 . If no edges have weight 1 , then the graph is bipartite.

When an edge is inserted, determine if it is odd or even by using the dynamic tree data structure of $F$, and give it weight 1 or 0 accordingly.

When an edge is deleted, if it is a tree edge, and if it is replaced with an odd edge (because there are no weight 0 replacements), remove the odd edge and find its next replacement, remove that, etc. until there are no more replacements. Then relabel the replacement edges as even and add them back to $G$.

Correctness: When an edge is inserted, the algorithm determines if it is even or odd. If an edge is deleted, we replace it by an even edge if possible. This does not affect the parity of the remaining edges. If no even replacement edge exists, but an odd replacement edge, the parity of every edge on the cut changes. However, since no even edge exists on the cut, it suffices to make all odd edges into even edges.

Analysis of Running Time: An even edge never becomes odd. Thus, the weight of an edge changes at most once, which shows that an insertion of an edge causes the edge to be added to the data structure at most once with weight 1 and at most once with weight 0 . The deletion of an edge leads to the removal of the edge from the data structure. Thus, the amortized expected update time is $O\left(\log ^{3} n\right)$.

ACKNOWLEDGMENTS We are thankful for David Alberts for comments on the presentation. 


\section{REFERENCES}

Alberts, D., And Henzinger, M. R. 1998. Average case analysis of dynamic graph algorithms. Algorithmica 20, 1, 32-60.

Eppstein, D., Galil, Z., Italiano, G. F., and Nissenzweig, A. 1997. Sparsification-A technique for speeding up dynamic graph algorithms. J. ACM 44, 5 (Sept.), 669-696.

Eppstein, D., Galil, Z., Italiano, G. F., and Spencer, T. 1996. Separator based sparsification for dynamic planar graph algorithms. J. Comput. Syst. Sci. 52, 1, 3-27.

Eppstein, D., Italiano, G. F., Tamassia, R., Tarjan, R. E., Westbrook, J., and Yung, M. 1992. Maintenance of a minimum spanning forest in a dynamic planar graph. In J. Algorithms 13, 33-54.

Even, S., And Shiloach, Y. 1981. An on-line edge-deletion problem. J. ACM 28, 1 (Jan.), 1-4.

Frederickson, G. N. 1985. Data structures for on-line updating of minimum spanning trees. SIAM J. Comput. 14, 781-798.

Frederickson, G. N. 1997. Ambivalent Data Structures for Dynamic 2-Edge-connectivity and $k$ Smallest Spanning Trees. SIAM J. Comput. 26, 2, 484-538.

Galil, Z., And Italiano, G. F. 1992. Fully dynamic algorithms for 2-edge connectivity. SIAM J. Comput. 21, 1047-1069.

Henzinger, M. R. 1995. Fully dynamic biconnectivity in graphs. Algorithmica 13, 503-538.

HENZINGER, M. R. 1999. Improved data structures for fully dynamic biconnectivity in graphs. SIAM J. Comput., to appear.

Henzinger, M. R. 1994. Fully dynamic cycle-equivalence in graphs. In Proceedings of the 35th Symposium on Foundations of Computer Science. IEEE Computer Society Press, Los Alamitos, Calif., pp. 744-755.

Henzinger, M. R., And Fredman, M. L. 1998. Lower bounds for fully dynamic connectivity problems in graphs. Algorithmica 22, 351-362.

Henzinger, M. R., And La Poutré, H. 1995. Sparse certificates for dynamic biconnectivity in graphs. In Proceedings of the 3rd European Symposium on Algorithms. Springer-Verlag, Berlin, Germany, pp. 171-184.

Henzinger, M. R., AND Thorup, M. 1997. Improved sampling with applications to dynamic graph algorithms. Rand. Struct. Algor. 11, 4, 369-379.

Holm, J., De Lichtenberg, K., And Thorup, M. 1998. Poly-logarithmic deterministic fullydynamic algorithms for connectivity, minimum spanning tree, 2-edge, and biconnectivity. In Proceedings of the 30th Symposium on Theory of Computing (Dallas, Tex., May 23-26). ACM, New York, pp. 79-89.

Miltersen, P. B., Subramanian, S., Vitter, J. S., and Tamassia, R. 1994. Complexity models for incremental computation. Theoret. Comput. Science 130, 203-236.

NAGAMOCHI, H., AND IBARAKI, T. 1992. Linear time algorithms for finding a sparse $k$-connected spanning subgraph of a $k$-connected graph. Algorithmica 7, 583-596.

Sleator, D. D., And Tarjan, R. E. 1983. A data structure for dynamic trees. J. Comput. Syst. Sci. 24, 362-381.

SPIRA, P. M., AND PAN, A. 1975. On finding and updating spanning trees and shortest paths. SIAM J. Comput. 4, 375-380.

TARJAn, R. E., AND Vishkin, U. 1985. An efficient parallel biconnectivity algorithm. SIAM J. Comput. 14, 862-874.

Westbrook, J., And Tarjan, R. E. 1989. Amortized analysis of algorithms for set union with backtracking. SIAM J. Comput. 18, 1-11.

RECEIVED NOVEMBER 1996; REVISED NOVEMBER 1998; ACCEPTED FEBRUARY 1999 